because of its action upon the trophic nerves. Dr. I Bemiss but echoes the opinion of all when he says "the great indication is to secure cinchonism as speedily as possible." And yet, we must say this cum grano salis. He who gives it as a routinist may kill his patient with the best antiperiodic in the pharmacopocia, for it is powerless to cure when permanent organic lesion keeps up the periodic action. In antiperiodic doses it equalizes vital force by promptly and powerfully impressing the central nervous system, thus relieving engorged and congested central organs. In toxic doses it preserves the restored balance. In antiphlogistic doses, even when there is organic change beyond its reach, it temporarily restores balance, arrests destructive metamorphoses and reduces pyrexila.

What has hygiene accomplished under the prevalent idea of callsation? Have any means been discovered to destroy the undiscovered entity-malaria? The drained marsh, until thoroughly dry, has only increased "palludal" fevers by increasing evaporation aud consequently intensifying diurnal and nocturnal alternations of temperature. Germicides and antiseptics are impracticable and useless.

The only effective, because the only rational hygiene, is found in avoiding exhausting labor during the heat of the day, replacing, as the chill of night approaches, damp clothing for such as is dry and warm, and slecping between comfortable blankets, keeping out of the chill and damp of night, or providing against it with proper clothing, and in avoiding all irregularities, and by healthful exercise and diet. By such precautions we may remove from the people the undefined dread of an indefinable malaria, rob the routinist of his ever-ready cry of malaria and its corollary, quinine, and feel as safe at the tropics as the poles; as safe on the swamp as on the mountain.

\section{THE NECESSITY OF ABDOMINAL SECTION, AS ILLUSTRATED BY AN UNUSUAL CASE OF OVARIOTOMY.'}

BY C. R. REED, M.D.,

of MIDDi.eport, OH16.

An ovariotomy to attract the attention of the profession at the present time, must be unique and have some characteristics of peculiar interest. To open the abdominal cavity as an exploratory operation for the purpose of making clear an obscure diagnosis, to remove an ovarian cyst or tumor, a diseased ovary or tubes, to stitch up a wounded viscus, and to remove foreign matter from the peritoneal cavity, is now of every day occurrence, and almost universally admitted to be legitimate surgery. The operation as first performed by McDowell to remove an ovarian cystic tumor when the health and life of the patient was endangered by mechanical pressure on adjacent organs or structures; or that of Battey of removing the ovaries to hasten the menopause, thereby arresting the monthly congestion of the pelvic organs; re-

\footnotetext{
' Read in the Section on Obstetrics, at the Thirty-Seventh Annua Mecting of the American Medical Association.
}

tarding the growth of tumors, checking hamorrhages and curing many diseases of the pelvic organs; and the exploratory sections as now advocated by Tait and others are so frequent as to almost cease to challenge attention. Without abdominal incisions and explorations the knowledge of pathological condition is often impossible; the diagnosis is obscure, the indications of treatment are uncertain, the patient does not receive the necessary treatment, the physician's reputation suffers and a post-nortem exam. ination reveals "what might have been." The success attending the operations, the necessity for which are made clear by exploratory incisions of the abdominal walls. is such as to deprive ovariotomy and kindred operations of their former terrors; to greatly enlarge the resources of the surgeon and to give new hope to the otherwise doomed patient.

The following case, I think, is a striking example of the necessity of abdominal incision and exploration without which its nature would no: have been known, the treatment would have been unsuccessful, the patient's life would have been a sacrifice to ignorance, and the grave would have kept the secret of its true pathology. As an illustration of the utter incfficiency of drugs in similar cases and the imperative necessity of bold and prompt surgical interference, no more striking instance, I believe, is on record. As a case of simple ovariotomy, after the diagnosis was made clear by abdominal section, there is nothing unique or unusual connected with it; but its history, the attending symptoms, the means used for relief give it, we think, an unusual interest. The symptoms during the few days, immediately preceding the operation, were such as had not before come under my ubservation in cases of ovarian cyst, neither had I seen similar symptoms described in the literature on the subject. The illness of the woman did not lead us to suspect an ovarian cystic tumor or any disease peculiar to her sex. I expected to find an entirely different pathological condition, and but for the exploratory incision, or an autopsy, the cause of the symptoms and the death of the patient, which I believe would have been inevitable, would still have remained a mystery; but the abdominal section at once nuade the diagnosis clear and the treatment plain.

In the afternoon of August 25, 1885, I was called in consultation with Dr. J. W. Lilly, of Pomeroy, Ohio, to see Mrs. B., married, 34 years of age, the mother of seven children, the younger two being twins, i 2 months old. She was then menstruating, having been regular the past three months. Dr. I.illy had called on me in the forenoon, gave me a history of the case, the symptoms and treatment, and requested me to see the patient with him. I gave an opinion then that nothing but laparotomy or an abdominal incision would reveal the true condition and indicate the treatment. Mrs. B. had been in good health up to within a few days prior to this date. The preceding four days she had vomited almost constantly, the last forty-eight hours it being stercoraceous. There was obstinate constipation, indicating obstruction of the bowels, cathartics, injections and local applications had been used without avail. 
Her pulse was $\mathbf{2 0}$ and weak, extremities cold, body covered with cold perspiration, strength greatly depressed from constant nausea and voniting; her ap-pęarance and general symptoms betokening rapid dissolution. Her mind being clear abdominal section was proposed as a possible means of relief, to which she and her husband readily consented. She was given a $1 / 2$ grain of morphia sulphas by subcutaneous injection, and Dr. J. H. Hysell was sent for to administer the aniesthetic. She was placed on the operating table, the abdomen laid bare, and washed with carbolized water, when it was discovered that the abdomen was enlarged and irregular which was attributed to frecal accumulation. She came promptly under the infiuence of the anesthetic, the A. C. E. mixture, when an incision was made in the linea alba, midway between umbilicus and pubes, four inches long; a tumor appearing at the opening, the incision was enlarged to eight inches, when it was found to be a cyst of the left ovary with adhesions to the abdominal walls and also to the descending colou. The former were readily broken up, the latter with difficulty, and these were believed to be the cause of the obstruction and constipation. There was general peritonitis, both of that portion lining the cavity and that investing the intestines, the tumor had a mottled appearance indicating congestion. The cxploring needle of a hypodermatic syringe was thrust in to the tumor, when the canula filled with a chocolate colored fluid. We had not come prepared to deal with an ovarian cyst; we had neither trocar, or hemostatic forceps, and were warned by the gentleman administering the aniesthetic that in the patient's depressed condition no time could be lost in sending for the necessary instruments, but the operation must be completed with what we had on hand. It is worthy of notice that no vomiting occurred after the incision was made, neither did it occur during convalescence. We determined to remove the tumors. After the adhesions were broken up the tumor was punctured with a scalpel and the fluid was conveyed away as best we could, by thrusting a rubber tube into the opening made by the knife. The pedicle was short and thick; it was transfixed by a double silk ligature, one end hanging from the lower angle of the wound for the purpose of drainage; the abdominal cavity was sponged dry, the incision closed by deep silk sutures and adhesive plaster. The patient rallied well from the anasthetic; her condition improving as soon as the nausea ceased; her bowels were spontaneously moved on the eighth day; convalescence was interrupted on the tenth day by a severe attack of capillary bronchitis, caused by the injudicious use of ice to lower the temperature. The ligatures on the pedicle came away on the fortieth day, and since that time she has had her usual good health; menstruated after the third month and is now two months enceinte.

The minor details of this case have been given in this paper because of its obscurity prior to abdom. inal section; the unusual symptoms attending it; the treatment prior to operation being based on an obscure diagnosis and therefore futile, and the diffi- culties of an operation in the absence of what are considered essential instruments. In considering the above case the following questions are suggested:

1. Was the ovarian cyst with its adhesions to the descending colon the cause of the obstruction of the bowels?, 2. Would the exploring needle have made the diagnosis clear without abdominal section? 3. With the probability of the patient dying under the operation would it have been better practice to have tapped with the trocar, and deferred the removal of the tumor? 4. As it has been shown by the large experience of I awson 'Tait and others that abdominal section and exploration is under aseptic treatment, attended with little or no mortal ty, is it not the imperative duty of the physician to open the abdomen in all cases of doubtful diagnosis where gencral treatment has failed to relieve the symptoms. 5. In cases of slight enlargement of the abdomen, attended with intestinal obstruction, as in this case, should the physician wait until symptoms of ap. proaching dislocation occur before resorting to abdominal section? And should the operation be performed as a means of diagnosis only, when the symptoms are not urgent?

\section{FRACTURES OF THE FEMUR IN CHILDREN,} Treated by Bryant's Method of Vertioal Extension.' BY J. M. BARTON, A.M., M.1).,

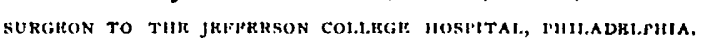

The treatment of fractures of the femur in children has always been attended with great difficulty. The fragments are so short they are not readily grasped by the dressings, and the thigh is usually so comparatively large and soft that the extremities of the fragments are with difficulty maintilined in apposition. None but the most firm and secure dressings will be retained, as all the efforts of the sufferer are directed towards their removal. The dressings are soon soiled by the discharges from the bladder and bowels, the frequent renewals interfering with the process of repair. The displacement of the dressings by the patient, causing injurious pressure, and the uncertainty which the surgeon feels concerning the condition of the limb, necessitate frequent examinations, especially when the child shows evidences of discomfort.

The disappointment occasioned by these dressings is perhaps best shown by the fact that in St. Bartholomew's Hospital Reports for 1867 , we find that Messrs. Paget and Callender report that they treat many cases of fracture of the thigh in children negatively, without splints, all apparatus being dispensed with, "the child being laid on a firm bed with the broken limb, after setting it, bent at the hip and knee and laid on its outer side."

The plaster dressing is a valuable one in these fractures, and as usually applied, with short splints around the femur and a long splint reaching from the axilla to beluw the foot, thoroughly covered in with plaster bandages, and either varnished or covered with oiled

1 Rend in the Section on Surgery, at the Thirty-Seventh Annual Mecting of the American Medical Association. 